\title{
Philosophiques
}

\section{Réformisme pénal et reponsabilité : une étude philosophique}

\section{François Blais}

Volume 16, numéro 2, automne 1989

URI : https://id.erudit.org/iderudit/027082ar

DOI : https://doi.org/10.7202/027082ar

Aller au sommaire du numéro

Éditeur(s)

Société de philosophie du Québec

ISSN

0316-2923 (imprimé)

1492-1391 (numérique)

Découvrir la revue

Citer cet article

Blais, F. (1989). Réformisme pénal et reponsabilité : une étude philosophique. Philosophiques, 16(2), 293-325. https://doi.org/10.7202/027082ar

\section{Résumé de l'article}

Cet article a comme principal objectif d'effectuer un bilan critique de certaines thèses du réformisme pénal (doctrine sociale privilégiant une approche thérapeutique de la criminalité par rapport à une approche punitive). La perspective retenue est celle de la responsabilité dans les différentes constructions théoriques du projet réformiste (médico-légale, déterministe et de " responsabilité stricte »). Je rendrai compte des thèses respectives sur le sujet et je ferai, en conclusion, certains commentaires critiques sur les difficultés pour ces approches réformistes de la responsabilité d'offrir un point de vue cohérent et moralement acceptable de la question. 


\title{
RÉFORMISME PÉNAL ET RESPONSABILITÉ : UNE ÉTUDE PHILOSOPHIQUE *
}

\author{
par François Blais
}

\begin{abstract}
RÉSUMÉ. Cet article a comme principal objectif d'effectuer un bilan critique de certaines thèses du réformisme pénal (doctrine sociale privilégiant une approche thérapeutique de la criminalité par rapport à une approche punitive). La perspective retenue est celle de la responsabilité dans les différentes constructions théoriques du projet réformiste (médico-légale, déterministe et de « responsabilité stricte »). Je rendrai compte des thèses respectives sur le sujet et je ferai, en conclusion, certains commentaires critiques sur les difficultés pour ces approches réformistes de la responsabilité d'offrir un point de vue cohérent et moralement acceptable de la question.
\end{abstract}

ABSTRACT. The main objective of this paper is to criticize some propositions of the social doctrine of penal reformism (the social doctrine which favors a therapeutic attitude toward a criminal rather than a punitive one). The perspective adopted is that of the responsability in the different theoritical constructions of the penal reformism doctrine (medico-legal, determinist and the strict-liability). I will explicite some thesis on the subject and I will do, in my conclusion, some critical commentaries on the difficulties that these three versions of penal reformism face when they have to give a coherent and morally acceptable point of view of the problem of responsibility.

Mais celui qui réfléchit quand il veut infliger une correction, son motif n'est pas de tirer vengeance de l'injustice passée, car il ne saurait faire que ne se soit pas produit ce qui a été accompli ; c'est au contraire l'avenir qu'il a en vue, pour éviter de nouvelles injustices, et de la part de ce même individu, et de la part de quiconque aura été témoin de la correction infligée.

(Platon, Protagoras, 324b)

* Je tiens à remercier Pierre-Yves Bonin, J. Nicolas Kaufmann et Georges Leroux pour leurs commentaires sur le contenu de cet article. Ces commentaires mont permis d'éviter diverses méprises. 


\section{PRÉSENTATION}

Le réformisme pénal dont il sera question dans cet article se présente comme une doctrine sociale privilégiant le traitement thérapeutique de la criminalité par rapport à la punition conventionnelle. Cette prédilection pour ce type d'intervention auprès du criminel demeure une question de degré et d'opportunité variable dans l'ensemble du discours réformiste mais elle s'établit cependant à partir de présupposés communs qui renvoient tous à certaines croyances sur la nature de la délinquance et sur le pouvoir de la science, particulièrement des sciences humaines, d'agir sur la criminalité et plus spécialement d'améliorer le potentiel réhabilitatif de la délinquance en général. Ces croyances des réformistes sont, pour l'essentiel, (i) que la plupart des délinquants peuvent ou encore plus simplement doivent être considérés comme malades ; (ii) qu'une approche thérapeutique est en général celle qui offre le plus d'opportunité de réhabilitation des criminels (si on la compare à l'approche punitive); (iii) que les sciences humaines sont en mesure d'apporter un éclairage nouveau sur les causes de la criminalité, sur la façon de la contrôler, dans le but ultime de proposer des interventions efficaces et réhabilitatives pour l'ensemble des individus qui forment la population carcérale ${ }^{1}$.

Les réformistes en viennent ainsi à une remise en question assez radicale du droit criminel, et plus particulièrement des pratiques et motifs habituels liés à la décision de la sentence (sentencing), tel qu'il se pratique dans la majorité des sociétés occidentales. Ces critiques portent essentiellement (i) sur le caractère inadéquat de la punition vue comme « just merit» et sur « l'exemplarité » de la punition; (ii) sur l'inefficacité, l'aberration et le gaspillage humain des sentences proportionnelles au délit, et sur

1. La litréracure sur le réformisme pénal abonde. Parmi les ouvrages considérés comme des “classiques " on pourra consulter : F. Alexander er $\mathrm{H}$. Staub, The Criminal, the Jugde and the Public, Glencoe: Free Press, 1956; J. Hall, General Principles of Criminal Law, Indianapolis : Bobbs-Merrill, 1969 (seconde édition), particulièrement le chap. 13; H. KLARE (ed.) Changing Concepts of Crime and Its Treatment, Oxford, Toronto : Pergamon Press, 1966; K. Menninger, The Crime of Punishment, N.Y. Viking Press, 1968 ; G. ZII.BOORG, The Psychology of the Criminal Act and Punishment, N.Y.: Harcourt Brace, 1954. On pourra aussi consulter des ouvrages plus récents comme N. WALKER, Punishment, Danger and Stigma, The morality of criminal justice, Totowa, N.J : Barnes \& Noble, 1980 et J.C. GoodWIN, Insanity and the Criminal, N.Y. Da Capo Press, 1981. 
l'urgence de lui préférer une sentence «individualisée » et réhabilitative; (iii) sur l'importance d'aborder les problèmes de moralité publique avec une approche scientifique plutôt que morale (les réformistes sont pour la plupart des praticiens/chercheurs des sciences humaines : psychiatres, psychologues ou criminologues) ${ }^{2}$.

Le but de cet article n'est pas de dresser les origines historiques du réformisme pénal (en philosophie on en retrouve déjà des traces chez Platon) mais plutôt de traiter des rapports que peut entretenir cette doctrine avec la philosophie, plus précisément avec la philosophie du droit et la philosophie de la responsabilité ${ }^{3}$. Je prendrai donc une certaine distance par rapport aux considérations qui furent traditionnellement celles qu'échangèrent la philosophie et le réformisme pénal. Ces considérations en philosophie se concentrent habituellement autour des débats entourant la justification de la punition (les réformistes sont foncièrement utilitaristes dans leur façon d'envisager cette rationalisation) ${ }^{4}$. La visée de cet

2. Sur l'association criminalité-maladie on pourra lire C. Darrow, Crime: It's Cause and Treatment, N.J.: Patterson and Smith, 1972 (première édition: 1922) et Bernard C. GLuECK, "Changing Concepts in Forensic Psychiatry ", Journal of Criminal Law, Criminology and Political Science, July-August, 1954.

Sur le problème spécifique de "l'injustice » de la justice, voir deux autres «classiques» du réformisme pénal: Bernard SHAw, The Crime of Imprisonment, Westport: Greenwood Press, 1969 (première édition 1946) et K. MENNINGER, op.cit. (particulièrement le chap. 3 : «Crimes against Criminals»).

Sur la question plus générale d'aborder les problèmes humains à l'aide des outils que la science est en mesure de nous procurer voir un personnage illustre du réformisme pénal : B. WOOTTON dans son Testament for Social Science : an Essay in the Application of Scientific Method to Human Problems, London: George Allen \& Unwin Ltd, 1950 et pour une critique de la thèse de Wootton voir Vinit HaKSAR: "A Scientific Morality?", Philosophy, vol. 42 (juillet 1967), pp. 245-65.

3. Sur l'histoire du réformisme pénal on pourra lire : N. WALKER, Crime and Insanity in England, Edinburgh : Edinburgh University Press, 1968 ; R. SMITH : Trial by Medecine. Insanity and Responsibility in Victorian Trials, Edinburgh : Edinburgh University Press, 1981 ; David GarLAND : Punishment and Welfare. A History of Penal Strategies, Aldershot, Hants, Eng. : Gower, 1985.

4. La préoccupation majeure de la philosophie en rapport à la punition fut et demeure celle de sa justification morale. Traditionnellement, on a opposé deux camps dans la littérature sur cette question: le rétributivisme et l'utilitarisme avec un nombre impressionnant de positions intermédiaires. La littérature philosophique sur la punition, ses définitions et les débats qui y sont impliqués est trop importante pour penser en faire mention ici. Je me permettrai néanmoins de mentionner trois articles importants qui font ceuvre de référence : J.D. MAsBotT, «Punishment », Mind, vol. 48 (1939), Pp. 152-67; A. FLEW, "The Justification of Punishment», Philosophy, vol 39, no 3 (octobre 1954), pp. 291-307 ; S.I. BENN : «An Approach to the Problem of Punishment ", Philosophy, 33 (1958), pp. 325-41. 
article n'est donc pas celle d'une justification ou d'une disqualification de l'ensemble des thèses réformistes. Il se veut plutôt une contribution à une discussion plus circonscrite qui conserve une certaine autonomie et donc tout son intérêt par rapport à la question de la justification morale de l'ensemble du projet réformiste. Il s'agit d'étudier la façon avec laquelle les réformistes traversent et réussissent à inflécbir le problème crucial de la responsabilité dans le droit. Je défendrai la thèse que l'attribution de la responsabilité dans le processus judiciaire est une préoccupation centrale de tout discours réformiste et que les réformistes, tout en défendant des positions fort différentes sur la question, n'ont pas d'autres choix que de postuler et de défendre certaines thèses fondamentales à l'égard de la responsabilité criminelle. Le but de cet article est en premier lieu de faire un bilan critique de ces différentes thèses réformistes. J'envisage ainsi de distinguer trois approches réformistes qui se différencient par l'interprétation qu'elles font d'une théorie de la responsabilité et par la place qu'elles lui confèrent à l'intérieur du droit (médico-légale, déterministe et de "responsabilité stricte »). Je soulèverai, en conclusion, les principales difficultés et confusions que ces doctrines ont tendance à entretenir à l'égard de la notion de responsabilité.

\section{LA VERSION MÉDICO-LÉGALE DU RÉFORMISME PÉNAL}

Cette première version du réformisme pénal tire ses origines de la psychiarrie et plus particulièrement de l'un de ses secteurs d'activités : la psychiatrie médico-légale. La notion d'insanité criminelle y joue un rôle de premier plan. Elle invoque la nonresponsabilité des actions tout en justifiant le remplacement de la punition par des méthodes thérapeutiques. J'accorderai donc mon attention, dans cette première partie, à l'étude du concept d'insanité criminelle (aux implications procédurales nouvelles de celui-ci pour le droit criminel) et plus particulièrement à la controverse qu'il a suscitée dans la pénologie moderne, controverse alimentée entre autres par les réformistes. Mais, avant d'aborder ce problème, je caractériserai grossièrement le concept de «responsabilité » tel qu'il est utilisé en droit afin de mieux cerner les implications immédiates de la critique réformiste. 


\subsection{Responsabilité et droit : le «mens rea»}

Depuis toujours, le droit criminel anglo-saxon a formalisé le principe voulant qu'une personne, pour être désignée coupable d'un crime, doit aussi être reconnue responsable. Cette délicate question de l'attribution de la responsabilité criminelle s'effectue à partir de conditions qu'on retrouve énoncées à l'intérieur de la doctrine du « mens rea » ${ }^{5}$. Cette doctrine reconnaît habituellement trois conditions à l'imputabilité : capacité, opportunité et connaissance ${ }^{6}$.

Ces trois conditions, auxquelles on réfère pour déterminer la responsabilité d'une personne pour un crime, sont une façon d'examiner la présence ou l'absence de certains « éléments mentaux » lors du délit. On dira par exemple qu'une personne qui ne pouvait connaître la portée de ses gestes ne peut être reconnue responsable de ses actions. Qu'une autre personne qui n'avait pas le pouvoir de changer quoi que ce soit au fil des événements ne peut, elle aussi, être tenue responsable des conséquences de ses actions. Il en est de même pour un agent qui, victime de la contrainte ou de pressions exercées contre lui, pose certains gestes condamnables contre son gré?

Ces composantes du «mens rea», malgré toutes les critiques qu'on peut leur faire et les questions qu'elles soulèvent encore aujourd'hui, n'en sont pas moins reconnues la plupart du temps

5. "Acrus non est reus nisi mens sit rea".

6. Pour une lecture plus approfondie sur le sujet: J.F. STEPHEN : A History of the Criminal Law, N.Y. : Burt Franklin 1973 (première édition 1883). Lire particulièrement le chapitre 18: «Criminal Responsibility». Il n'y a pas consensus sur les conditions de validité que devrait comprendre le «mens rea». Celles que je présente sont celles retenues par H.L.A. HaRT dans "Acts of Will and Responsibility », tirés de Punishment and Responsibility. Essays in the Pbilosophy of Law, Oxford : Oxford University Press, 1968, p. 90-112. Stephen, pour sa part, retient 4 conditions essentielles qui divergent un peu de celles de Hart et qu'il désigne comme: age, connaissance, intention et minimum de contrôle musculaire. La condition d'àge est évidente et on peut croire que la définition de Hart l'embrasse mais celle d'intentionnalité fait problème pour ce dernier. Il lui préfère la notion d'opportunité. Voir à ce propos sa critique dans « Acts of Will... 》.

7. Ce qui correspond en gros aux conditions invalidantes de la responsabilité chez Aristote : la contrainte et l'ignorance. Il existe des délits où, par exception, la présence de certains de ces «éléments mentaux» n'est pas requise pour que soit reconnue la responsabilité. Ce sont les cas de négligence criminelle et de “responsabilité stricte», nous reviendrons plus loin sur ce problème. 
comme des indices clairs d'une certaine maturité de notre pratique du droit tout comme de la façon dont nous nous y prenons généralement pour former nos jugements vis-à-vis de nos pairs. Les raisons motivant leur usage sont donc très importantes. Il s'agit en substance de dépasser le cadre étroit d'une enquête qui se limiterait à un simple examen des faits et des comportements extérieurs à l'origine d'un état de choses dans le monde (ex. : vol, meurtre, fraude). En effet, le recours au «mens rea» nous permet de poser le problème de l'imputabilité non seulement en termes de causalité opérante entre un événement et d'autres constituant sa prolongation à l'intérieur d'une séquence, mais aussi en termes d'actions bumaines (où des facteurs comme l'intentionnalité ou l'opportunité peuvent devenir déterminants). Nous avons affaire ici à deux types d'enquêtes de l'analyse causale: l'une qui se rapporte à des événements extérieurs à l'individu mais dont il peut être reconnu comme l'agent initiateur, l'autre à des événements internes qui expliquent sa conduite. On ne reconnaît donc pas la responsabilité à la simple existence de certains liens entre un agent et les conséquences de ses actions mais aussi au contexte d'émergence des actions humaines à l'origine de la situation. Le recours au «mens rea» oblige à ce qu'on tienne compte de la présence ou de l'absence de certains «éléments mentaux » chez tout présumé coupable, car ce qui nous permet de distinguer un meurtre volontaire d'un meurtre involontaire n'est pas uniquement l'enquête des faits matériels entourant le meurtre mais aussi, pour prendre un exemple, une étude des intentions de l'inculpé qui permet en bout de ligne de qualifier l'action « $\mathrm{X}$ a tué $\mathrm{Y}$ » de façon différente. Cette dernière investigation est tout aussi importante que la première et elle permet d'attribuer la responsabilité d'un délit à une ou des personnes en respectant des modalités qui fondent l'ensemble de nos discours moral et juridique. Sous ces modalités, être reconnu comme étant la cause d'une infraction (au sens de résultats, effets, conséquences) ne justifie pas que l'on en soit tenu automatiquement responsable (au sens d'être candidat à la punition [ «liable to punisbment»]). D'autres conditions comme celles reconnues par le «mens rea» s'appliquent ${ }^{8}$.

8. Il ne faut pas que les expressions utilisées dans ce passage puissent porter à confusion. Quand j'affirme que l'attribution de la responsabilité exige une enquête différente de celle qui ne s'attarde qu'aux faits extérieurs ou observables, je ne veux pas dire que les énoncés d'action renvoient à de l'inobservable. Je cherche plutôt à distinguer la nature 


\subsection{L'insanité criminelle : la règle de M'Naghten}

Dans la lignée et l'esprit du "mens rea ", le droit anglo-saxon a reconnu le privilège de l'immunité pour certains inculpés chez qui on peut faire la preuve de la présence de certains troubles mentaux. La non-imputabilité en question est valable même si la situation entourant le crime satisfait, du moins à un premier niveau, les conditions du «mens rea ». Ainsi, pour une personne à qui incomberait normalement la responsabilité d'un crime, il est possible d'invoquer une situation de non-imputabilité (complète ou partielle) si la défense peut faire la preuve de certaines incapacités mentales tombant sous le coup des critères de la règle de M'Naghten. Cette règle fut adoptée en 1843 en Angleterre suite à un procès devenu célèbre et qui devait soulager Daniel M'Naghten de la responsabilité du meurtre de Sir Robert Peel, secrétaire privé de la couronne. Elle se formule ainsi :

...to establish a defence on the ground of insanity it must be clearly proved that, at the time of committing the act, the party accused was laboring under such a defect of a reason, from disease of the mind, as not to know the nature and quality of the act he was doing; or, if he did know it, that he did not know he was doing what was wrong?

La règle de M'Naghten aura comme premier effet, on le comprendra, de cautionner directement les interventions de la psychiatrie-légale à titre de témoin expert dans certaines causes où la défense tente de démontrer que l'inculpé n'avait pas la connaissance complète de ce qu'il faisait au moment où il agissait. Elle constitue donc un premier moment historique pour le sujet qui nous intéresse. En effet, la défense qui réussit à faire la preuve que son client doit être tenu non responsable pour raison d'insanité, se

de ceux-ci. Par ailleurs, je n'entretiendrai pas dans cet article la distinction entre base causale de l'attribution de responsabilité (lorsque la performance d'un agent $\mathrm{X}$ est la cause d'un événement $A$ ) er base non causale de l'attribution de responsabilité (lorsqu'un autre individu Y est dans une situation qui lui permet [ou l'oblige] de blâmer ou encore de récompenser $\mathrm{X}$ pour avoir performé $\mathrm{A}$ ). Cette distinction, quoi qu'importante dans les discussions philosophiques et légales sur la nature du concept de responsabilité n'amène pas d'éclairage important sur la problématique à l'étude. Pour plus d'information sur cette question, on pourra lire H.L.A. HarT et T. HonOrÉ, Causation in the Law, Oxford: Clarendon Press, 1959 ( $2^{e}$ édition: 1985). Particulièrement le chapitre III de la section I portant sur l'attribution causale de la responsabilité.

9. Tel que cité dans N. WALKER, Crime and Insanity in England, op. cit., p. 100 
retrouve habituellement à en faire du même coup un candidat à l'incarcération dans un asile, pour une période indéterminée, dans le but de suivre un traitement visant sa réhabilitation éventuelle ${ }^{10}$.

\subsection{La critique de la nature intellectualiste de la règle : la première tentative réformiste}

J. Feinberg ${ }^{11}$ dans un article consacré à la notion d'insanité mentale a montré que la règle de $\mathrm{M}^{\prime} \mathrm{N}$ aghten est en continuité avec l'esprit du "mens rea» (X ne peut être tenu responsable s'il ne savait pas ce qu'il faisait). La règle, selon lui, témoigne d'une certaine évolution du droit, sans plus. Ainsi, on ne peut conclure qu'elle constitue en soi une ouverture conceptuelle importante et qui permettrait à une quelconque stratégie réformiste de prendre assise. Au contraire, c'est dans la critique de cette règle que la version médico-légale du réformisme trouvera tout son sens et cherchera à se définir.

La plus importante et la plus déterminante des critiques portées à l'endroit de la règle de M'Naghten par la psychiatrie médico-légale a trait à son caractère intellectualiste. Les « maladies de l'esprit » que la règle reconnaît comme étant en mesure de limiter la responsabilité de l'agent sont des maladies qui entravent, au moment du délit, la connaissance de la nature du ou des gestes posés. Il ne s'agit donc pas de maladies pouvant troubler d'autres constituantes de l'agir humain comme la volonté par exemple, bien qu'il soit possible, croit-on, de faire la preuve que la nature de certaines maladies peuvent contraindre ceux qui en souffrent à des actions qu'ils n'auraient pas commises en temps normal. La règle met uniquement l'emphase sur la connaissance et sur la compréhension de nos actions, sans considérer d'autres facteurs de l'action humaine pourtant tout aussi déterminants, affirment ces psychiatres. La règle de $\mathrm{M}$ 'Naghten nous amènerait à une définition beaucoup trop restrictive de l'insanité criminelle. La base fondamentalement intellectualiste sur laquelle s'appuierait la règle exclut des groupes

10. Daniel M'Naghten fut interné en 1843 à l'hôpital de Bethleem jusqu'en 1846. Il fut a lors transféré à la Broadmoor Institution, une institution pour les « fous criminels » où il mourut en 1865 .

11. "What is so Special About Mental Illness?" dans Doing and Deserving : Essays in the Theory of Responsibility, Princeron : Princeton University Press, 1970. 
d'individus souffrant de sérieux problèmes psychiatriques (kleptomanie, compulsivité, exhibitionnisme, fétichisme, etc.) ${ }^{12}$. Ces problèmes, disent les psychiatres, entravent pourtant la liberté de plusieurs et leur font poser des gestes qu'ils ne commettraient pas en temps normal, cela, bien qu'ils en connaissaient la portée. Un individu reconnu comme souffrant d'une maladie mentale par la psychiatrie officielle doit faire la preuve de son ignorance devant la nature des gestes qu'il posait à ce moment s'il veut se prévaloir de l'immunité que la règle lui garantit. La gravité de sa maladie n'a donc rien à voir avec ce test de non-responsabilité si on ne peut faire la preuve qu'elle influençait la connaissance de ses actions au moment du délit ${ }^{13}$.

Intuitivement ou encore avec l'appui d'informations tirées des sciences du comportement, les praticiens de la psychiatrie mesuraient les limites de cette définition de l'insanité criminelle. Cette dernière leur paraissait nettement insuffisante pour rendre compte de la multitude de cas de contrevenants qui ne pouvaient et ne devaient pas être reconnus responsables de leurs actions. La British Medical Association, une des principales opposantes à la règle de M'Naghten, formula son désaccord ainsi :

It takes account only of the cognitive faculties and is therefore based on an antiquated and outworn conception of mental disease, which is now recognized as involving morbid emotional changes and not solely, nor even necessarily, an impairment of intellectual powers ${ }^{14}$.

On assiste à plusieurs tentatives de propositions de réforme dès le début du $20^{e}$ siècle afin de résoudre la soi-disant impasse laissée par la règle de M'Naghten. Toutes ces propositions originaient, il va sans dire, de ce courant réformiste et des insatisfactions ressenties à l'égard de l'application de la règle. Je ne passerai pas

12. Il ne faudrait pas commettre d'erreur ici sur le sens à donner à « intellectualiste » qui, en philosophie, a une connotation fort différente du contexte péjoratif dans lequel ces réformistes tentent de l'inscrire à l'intérieur de ce passage. «L'intellectualisme » dont ii est question ici a un sens réduit et plus spécifiquement cognitiviste.

13. On trouvera un récit des débats entourant la transformation de la règle de M'Naghten dans B. Wootron, Social Science and Social Pathology, London: George Allen \& Unwin, 1959 (aux chap. VII : "Social Parhology and the Concepts of Mental Health and Mental Illness» et VIII : "Mental Disorder and the Problem of Moral and Criminal Responsibility ") et dans J. FeInBERG, op. cit.

14. Citation reprise de B. WOOTTON, ibid., pp. 231-32. 
en revue les différentes formulations d'amendement à la règle qui ont été proposées tout au long d'un débat qui n'a, de toute façon, pas encore trouvé son achèvement. En substance, les contenus des revendications de réformes attendues se ressemblent tous. Le but est d'admettre une condition nouvelle de non-responsabilité pour cause d'insanité criminelle: une maladie de l'esprit pouvant influencer la volonté des individus. La formulation la plus célèbre est celle de la British Medical Association proposée à la Royal Commission on Capital Punishment (1953):

(i) a defect of reason such that he did not know (a) the nature and quality of the act he was doing, or (if he did know this) (b) that he was doing what was wrong; or

(ii) a disorder of emotion such that, wbile appreciating the nature and quality of the act, and that it was wrong, be did not possess sufficient power to prevent himself from committing it ${ }^{15}$.

Cette formulation peut parfaitement tenir lieu d'énoncé typique des revendications d'amendement de la psychiatrie médicolégale à l'égard de la règle de M'Naghten. Ces médecins réformistes désirent que le droit reconnaisse que la responsabilité des actions d'un individu ne puisse être en cause lorsque celui-ci est ignorant de la nature ou de la portée de ses gestes (proposition (i)), mais aussi, lorsqu'il est reconnu qu'une pathologie de l'esprit le privait du pouvoir nécessaire (volonté) pour se contrôler (proposition (ii)). Il s'agit, en d'autres mots, de désigner certains « désordres de la volonté » comme étant des facteurs pouvant influencer nos jugements de responsabilité à l'égard d'autrui. En faisant une proposition du genre, ces médecins prétendent du même coup pouvoir munir la pratique du droit d'un test de non-responsabilité pour cause d'aliénation mentale suffisamment adéquat pour rendre enfin compte de la multitude des cas de contrevenants souffrant de maladie mentale.

Le contenu des revendications médicales indique bien sûr que c'est à la psychiatrie que reviendra la tâche d'établir les critères à partir desquels la capacité d'agir d'un criminel pourra être reconnue

15. Citation reprise de B. Wootton, ibid., p. 232. Je souligne. 
suffisamment défectueuse ou insuffisante pour le départir de la responsabilité de ses gestes. Ce sera à la psychiatrie de faire la preuve de ces insuffisances en s'appuyant sur des modèles théoriques et des données scientifiquement valables (observables et objectives). Le but ultime étant d'appuyer les jugements de non-imputabilité des candidats à l'insanité criminelle sur des considérations scientifiques. La façon d'y parvenir est de changer, partiellement du moins, notre conception de la responsabilité.

\subsection{Conséquences immédiates de la reformulation de la règle}

Les conséquences d'une telle proposition sont majeures. La plus importante d'entre elles consiste à transposer les discussions entourant l'imputabilité d'une problématique de connaissance (aspects cognitifs de la responsabilité) à une problématique de capacité (aspects conatifs). Cette nouvelle problématique sousentend que l'on s'attarde maintenant et plus que jamais à la capacité pour un individu de se conformer à une règle sociale et qu'on puisse rendre compte ultimement de cette capacité en la mesurant, en l'évaluant ou en la comparant.

L'incapacité de se conformer à une règle devient donc sous l'influence d'un certain courant psychiatrique du $20^{e}$ siècle la définition standard de la notion de non-responsabilité pour cause d'insanité criminelle. Cette nouvelle notion de non-responsabilité implique la possibilité pour les sciences humaines d'intervenir sur les discussions concernant l'imputabilité. Cela suppose aussi que l'action humaine puisse, ultimement, être identifiée à ses causes internes mais observables, et que l'attribution de la responsabilité découle d'un examen minutieux de l'enchaînement de ces causes entre elles. Cette perspective de la responsabilité emprunte ou s'associe à la science à partir de deux postulats fondamentaux :

(i) La notion de degré qui est sous-entendue dans la définition de la responsabilité comme capacité de se conformer à une règle. Une notion indispensable à la recherche scientifique et au caractère observable des «data» scientifiques, une notion qui invite à la mesure et à la comparaison. Dans le cas qui nous intéresse, une notion pri- 
mordiale pour que s'effectue l'évaluation entre une « force irrésistible » et une «force» qui ne le serait pas ${ }^{16}$.

(ii) Une conception causale de l'action humaine qui suppose que le comportement humain est propre à l'explication causale et susceptible d'être décomposée en faits observables, dans tous ses moments, de sa cause à son aboutissement. Chaque composante référant à de l'observable logiquement indépendant mais causalement lié.

En d'autres mots, la psychiatrie médico-légale tente de créer un espace épistémologique nouveau. Ce que l'on cherche à substituer en donnant une définition de la responsabilité comme capacité à se conformer à une règle, c'est une opportunité pour les sciences humaines d'établir la responsabilité d'une personne à partir de certaines données et de certains faits. C'est la croyance ferme que parmi les faits qui nous permettent habituellement de déterminer la responsabilité d'un agent, il s'en trouve de nouveaux qui peuvent être mis en lumière par les sciences. Car la question se pose pour l'ensemble des sciences humaines: comment pourrat-on mesurer le comportement d'une personne et faire la preuve que, dans certains cas, il lui était impossible de résister à la tentation, à la force intérieure le dominant, etc. ? "L'anti-intellectualisme » de ce projet réformiste repose sur cette conviction qu'il est possible de répondre à cette question fondamentale: «au moment de poser son geste, était-il possible pour $\mathrm{X}$ de s'en abstenir? »

\subsection{La version médico-légale : le constat d'un échec?}

Au chapitre 8 de Social Science and Social Pathology, Barbara Wootton ${ }^{17}$ fait un excellent examen critique des différentes tentatives des réformistes qui ont cherché à remplacer la nature intellectualiste

16. L'idée de capacité entre dans toute caractérisation de la responsabilité et n'est certainement pas un élément nouveau identifié par les réformistes. La différence dans ce cas-ci porte sur l'insistance mise sur cette partie du problème de l'attribution de la responsabilité et sur les informations nouvelles que l'on croit pouvoir mettre à jour pour mieux évaluer cette responsabilité.

17. Barbara Wootton est considérée comme l'une des principales représentantes du réformisme pénal. Nous verrons ultérieurement ses propres propositions à l'égard de la responsabilité et du droit, à la troisième partie du présent article. 
de la règle de $\mathrm{M}^{\prime}$ Naghten ${ }^{18}$. Elle conclut que la psychiatrie médicolégale a entretenu de nombreuses confusions autour de ce problème. Confusions qu'il lui apparaît important de démystifier. La plus fréquente de ces confusions consiste à fournir des explications causales de comportements en apparence complètes, mais sans offrir véritablement de critères permettant de différencier une action responsable d'une autre qui ne le serait pas. Une explication du comportement d'un individu, aussi objective et scientifique qu'elle puisse prétendre être, demeure une description de faits qui, à elle seule, n'est pas en mesure d'offrir ou de discréditer les conditions nécessaires de l'imputabilité ${ }^{19}$. Expliquer n'est pas disculper. On en arrive ainsi au problème central de cette approche réformiste qui, en plus d'offrir une explication scientifique, doit trouver un critère indépendant, une évidence observationnelle reconnue montrant l'incapacité pour des individus aux prises avec certains symptômes de se conformer à une règle.

Une autre confusion fréquente, nous dit Wootton, est de construire la justification théorique d'un modèle offrant une analyse de situations de non-responsabilité pour cause d'insanité sur les bases d'un raisonnement circulaire. Dans la majorité des cas, cela se présenterait des deux façons suivantes:

(i) soit que de la gravité du comportement délictueux on infère une maladie mentale qui à elle seule ne trouve de preuve de son existence qu'à l'intérieur de la gravité du comportement déjà mis en cause (ou de son extravagance, de son côté peu commun, disproportionné, etc.). La présence d'une maladie mentale entravant notre pouvoir d'agir doit pouvoir être reconnue, nous dit Wootton, à partir d'un critère indépendant du comportement qu'on veut justement faire reconnaître comme non responsable ;

18. Thomas Szasz fait lui aussi un examen critique de cette tentative au chap. 8 de La loi, la liberté et la psychiatrie, Paris, Payot, 1977.

19. Du muins c'est une thèse considérée comme centrale par HarT et HoNORÉ dans Causation in the Law, op. cit. La détermination de la responsabilité implique des éléments normatifs tout comme des éléments de causalité. Voir aussi J. Feinberg: "Problematic Responsibility in Law and Morals», Philosopby, vol. 71, 1962, pp. 340-350. 
(ii) soit qu'on insiste à outrance sur le caractère répétitif d'une offense en se trouvant justifié de demander un verdict de non-responsabilité du seul fait que l'offense fut répétée à maintes reprises, sans motif apparent et sans que l'aureur puisse apparemment s'en prévenir. Malheureusement, on ne peut conclure à l'irrésistibilité d'une « pulsion » du simple fait qu'un délinquant récidive. Il pourrait récidiver autant que faire se peut, nous ne pourrions jamais, rationnellement, en conclure qu'il n'avait aucun contrôle sur son comportement. La preuve reste à faire en fournissant un critère indépendant du comportement prétendument « compulsif $» . . .20$

Il est difficile de conclure définitivement à l'échec de la tentative d'identifier les seuils empiriques de la responsabilité, tellement l'entreprise est audacieuse et certainement complexe. Nous conviendrons pour le moment que la question reste ouverte et nous passerons à l'étude de la deuxième approche réformiste qui défendra des thèses nettement plus radicales à l'égard de la responsabilité, des thèses allant pour l'essentiel à l'encontre de la version médico-légale. Je reviendrai, en conclusion, sur un diagnostic plus précis et d’après moi plus déterminant des causes de ce présumé échec de la version médico-légale. La difficulté pour ces réformistes d'évaluer correctement la complexité du problème auquel ils s'attaquent y sera présentée comme nettement déterminante.

\section{LA VERSION DETTERMINISTE DU RÉFORMISME PÉNAL}

The determinist can make no distinction between the killing of a human being through criminal violence or through toxines of a tubercle bacillus ${ }^{21}$.

20. A ce même chapitre 8 de Social Science and Social Pathology, Barbara Wootton dresse un bilan des différentes tentatives de la psychiatrie qui lui ont paru échapper à ces erreurs de raisonnement et qui, de ce fait, lui apparaissent être des modèles théoriques plus acceptables. Il n'en demeure pas moins que tous (il y en a quatre) sont finalement jugés insatisfaisants par Wootton (voir les pages 237-45). Les raisons données seront : (i) le caractère normatif du critère utilisé (ex. : l'absence de motifs); (ii) la circularité des raisonnements impliqués (approche typiquement nosologique); (iii) le pragmatisme injustifiable (qui n'offre aucun critère vérifiable et indépendant); (iv) la non-vérifiabilité des présuppositions théoriques (la psychanalyse et la distinction conscient-inconscient).

21. A.A. Brill : «Determinism in Psychiatry and Psycho-Analysis », American Journal of Psychiatry, vol. 95 (1938), p. 597. 
Cette deuxième version du réformisme pénal essaie de se situer à l'encontre de la première sur certaines thèses importantes. Premièrement, la tentative d'établir des seuils de la responsabilité est abandonnée. De plus, la croyance selon laquelle la majorité de la population fait usage de rationalité lorsqu'elle agit est, quant à elle, totalement mise de côté (on ne peut, pour les déterministes, invoquer ni rationalisation ni autres motifs comme preuve de la liberté de nos actions). C'est donc toute la notion de responsabilité telle qu'on la perçoit habituellement qui est remise en cause. Ces réformistes adhèrent à une conception déterministe du comportement humain. À l'intérieur d'une telle approche, affirment-ils, le problème de la détermination de la responsabilité ne devrait plus se poser.

\subsection{La thèse déterministe : la seule réellement compatible avec le développement de la science}

No theory of mental medecine could develop without the working hypothesis of determinism... "free will» on which both law and religion are based, proves a heuristically sterile idea 22 .

Cette version réformiste réactive pour l'essentiel la doctrine philosophique du déterminisme et de la non-liberté de l'être humain. Son approche de la question se distingue toutefois de l'approche traditionnelle en philosophie du fait que pour étayer sa preuve, elle compte faire appel à certaines «évidences » qu'elle prétend tirer de la science.

Nous l'avons vu, les réformistes sont pour la plupart des praticiens des sciences humaines. Ce sont des hommes et des femmes qui estiment profitable d'emprunter à la science certains postulats fondamentaux pour les aider à réfléchir sur des problèmes sociaux comme la criminalité. Par ailleurs, on convient habituellement que les sciences exactes utilisent une notion huméenne de la causalité : c'est-à-dire un principe de causalité matérialiste où la relation entre cause et effet est nécessaire et où chaque rapport de cause à effet peut être dit déterminé par une loi plus générale. Les

22. E. SLATER : "The MNaghten Rules and Modern Conceptions of Criminal Responsibility ", British Medical Journal, Sept. 25, 1954. Citation reprise de B. WOOTTON : «Diminished Responsibility: A Layman's View», Law Quarterly Review, vol. 76 (1960), p. 282. 
déterministes en sciences humaines prétendent qu'il faille emprunter aux sciences naturelles le principe de causalité qu'elles utilisent (et qui implique la nécessité) si on veut en arriver à un projet de science heuristiquement valable, comme c'est le cas pour les sciences exactes. Le principe de causalité qui régit le monde des phénomènes doit donc être considéré identique à celui qui régit nos comportements. Une telle option est la seule qui permettrait à la recherche en sciences humaines, croit-on, de découvrir les lois universelles dont nos comportements sont les aboutissements naturels. Les réformistes déterministes adoptent cette conception de la causalité tout comme la règle déterministe qui semble être, pour eux du moins, son corollaire ${ }^{23}$. Cette idée rend mieux compte des réalités qu'ils côtoient dans leur pratique, particulièrement en ce qui a trait à l'étude des causes de la criminalité ${ }^{24}$.

\subsection{Les avantages de l'approche déterministe sur l'approche médico-légale}

«Man is predestined by anthropological, social and physical causes to violate the law... ${ }^{25}$. " Pour les tenants de la thèse déterministe, la liberté est considérée comme une illusion entretenue

23. Je dis "qui semble être son corollaire». Dans les faits, on peut convenir, et il est probablement préférable de le faire, de dissocier l'amalgame du principe de causalité universelle et du déterminisme. Le premier pourrait ainsi être considéré comme métaphysique ou heuristique alors que le deuxième pourrait être reconnu comme empirique et, pour cette raison, falsifiable. C'est du moins l'une des thèses principales de Peter van Inwagen dans An Essay on Free Will, Oxford: Clarendon Press, 1983. Voir particulièrement les pages $1-8$ ef $190-223$.

24. Pour ne nommer que quelques-uns des réformistes, ils sont nombreux, qui adhèrent à la thèse déterministe: L. Hall et S. GLueCK: Cases on Criminal Law and Its Enforcement, St-Paul, Minn., West Publishing Co., 1958; K. Menninger : op. cit.; J.C. GoOdwIN, op. cit.; Elliot SLATER : « The M Naghten Rules and Modern Conceptions Of Criminal Responsibility ", British Medical Journal, September 25, 1954. Ajoutons ici que ces réformistes déterministes commettent une fois encore l'erreur d'associer trop rapidement principe de causalité et principe du déterminisme, ce qui n'est pas du tout évident si l'on s'en tient à un autre débat en philosophie analytique entre les compatibilistes (l'acceptation du déterminisme ne met pas en cause la responsabilité) et les incompatibilistes (l'acceptation du déterminisme rend la responsabilité factice) en philosophie de l'action. Sur ce débat voir le survey de J.E. TILES, « Ability, possibility and responsibility", dans G. Floistad (ed.), Contemporary Philosophy (volume 3, Pbilosophy of Action), The Haghe: Martinus Nijhoff, 1982

25. Belbey: "Psychoanalysis and Crime», Journal. Crim. Psychopath, vol. 4 (1943), pp. 639-47. Citation reprise de J. HaLL: op. cit., p. 456. 
par la majorité des humains, une fausse croyance que le développement des sciences ne cesse de démystifier ${ }^{26}$. Il est possible, croientils, d'éviter l'impasse à laquelle les avaient conduites certaines présuppositions de la version médico-légale. L'une de ces présuppositions est la croyance en la prédisposition rationnelle de la majorité de la population (la majorité de la population est saine et on peut présumer de sa capacité à conformer ses comportements à un système de règles préétablies). Le test de responsabilité que la version médico-légale propose à l'intérieur de son programme ne touche que les cas de délinquance plus exceptionnels considérés comme des cas d'insanité criminelle. On ne retrouve pas une attitude aussi radicale que celle qui présupposerait que le criminel est par définition un malade ou que l'approche thérapeutique est la meilleure technique réhabilitative à encourager auprès de la majorité de la population carcérale. La version médico-légale est en général plus réservée quant à la possibilité d'élargir à l'ensemble de la population l'usage des techniques réhabilitatives.

Cependant, la conséquence de la présupposition en la prédisposition rationnelle de la majorité de la population, qui obligeait la version médico-légale à fournir un critère empirique non contestable permettant de distinguer un cas de non-responsabilité pour cause d'insanité d'une situation où l'on peut tenir une personne pleinement responsable de ses actions, n'a pas trouvé de formulation adéquate. La version médico-légale n'a pu élaborer un critère de l'insanité criminelle satisfaisant et avec des bases assez solides pour le rendre opérant à l'intérieur du processus judiciaire.

C'est sur ce point que les déterministes croient pouvoir prendre une longueur d'avance sur la version médico-légale. En effet, la même situation qui conduisit celle-ci à l'échec devient, chez les déterministes, un faux-problème ne méritant plus d'attention: "The phenomenon of the will like other natural phenomena are subject to natural laws and are determined by antecedents ; (..) responsibility, therefore, (...) does not exist scientifically in any

26. «L'homme autonome est un dispositif que l'on invoque pour expliquer ce que l'on ne peut expliquer autrement. Il s'est construit de nos ignorances, et à mesure que notre compréhension progresse, la matière dont il est fait s'évapore. » B.F. SKINNER: Par delà la liberté et la dignité. Montréal : Hurtubise, 1972, p. 243. 
case, sane or insane... ${ }^{27}$ " Les réformistes déterministes ne se retrouvent donc pas dans la situation difficile d'avoir à étayer leur propre position d'un modèle explicatif de non-responsabilité pour cause d'insanité, puisqu'ils n'adhèrent pas à un postulat comme la prédisposition à la rationalité de la majorité de la population ou, encore, à des notions comme la responsabilité diminuée ou la responsabilité tout court. $\mathrm{N}$ 'adhérant pas aux principaux postulats de l'approche médico-légale, ils s'épargnent les principales critiques qui lui ont été faites concernant la définition de l'insanité criminelle. La discrimination entre un acte pulsionnel et un acte qui ne le serait pas apparaît être un exercice dépourvu de sens pour un déterministe. Pour ce dernier, toute action est déterminée de façon nécessaire et il est impossible de présumer qu'un comportement puisse être plus déterminé qu'un autre. La théorie déterministe n'est pas en mesure, à strictement parler, d'évaluer la différence normative entre un crime prémédité et un accident de chasse. La responsabilité n'est pas donnée à l'observation.

\subsection{La thèse déterministe : un prétexte à l'intervention thérapeutique généralisée}

The very word « justice " irritates scientists. No surgeon expects to be asked if an operation for cancer is just or not. No doctor will be reproached on the grounds that the dose of penicillin he has prescribed is less or more than «justice» would stipulate. Behavioral scientists regard it as equally absurd to invoke the question to justice in deciding what to do with a woman who cannot resist her propensity to shoplift, or with a man who cannor repress an impulse to assault somebody. This sort of behaviour has to be controlled; it has to be stopped. This (to the scientist) is a matter of public safety and amicable coexistence, not of justice... ${ }^{28}$

Les réformistes déterministes ne croient pas que la science puisse être d'un quelconque secours au bon déroulement de la justice. Science et droit empruntent à des perspectives tout à fait différentes et par surcroît inconciliables. La première suppose l'existence de lois universelles régissant les faits du monde, la deuxième est normative et présuppose que nos actions sont la

27. G. Zll.Boorc, Mind, Medicine and Man, N.Y.: Hartcourt, Brace, 1943, p. 282. Je souligne.

28. K. MENNINGER, op. cit., p. 17. 
plupart du temps libres et qu'elles échappent, en partie du moins, aux lois conditionnant le monde extérieur. Cette inconciliabilité du droit et de la science n'empêche cependant pas les déterministes de porter un jugement sévère sur l'institution de la punition : "The criminal law represents "vengeance" (which) still functions but under disguise, namely, the disguise of deterrence... ${ }^{29}$ "

Là où les sciences peuvent être utiles, prétendent les réformistes, c'est dans la réforme des criminels ${ }^{30}$. Mais pour cela il faut favoriser, au détriment de l'approche punitive actuelle, des interventions thérapeutiques généralisées auprès de la population criminelle. La thèse déterministe, en niant la responsabilité, conduit le réformisme à une nouvelle interprétation de ce que devrait être le processus judiciaire, c'est-à-dire le processus par lequel un inculpé en vient à ètre acquitté ou condamné pour un crime. La tâche des juristes y est considérablement remise en question et les praticiens des sciences humaines y occupent désormais la place centrale.

Les réformistes réfèrent à des principes moraux différents des principes habituellement reconnus par les législateurs pour défendre leur système. Le problème central de l'institution de la punition, c'est son inefficacité. L'objectif d'une plus grande efficacité de nos institutions sociales devrait donc être reconnue dans l'avenir comme la valeur à promouvoir. Le recours à la science se révèle particulièrement une bonne façon d'augmenter notre pouvoir sur le monde, de le transformer afin qu'il réponde mieux à nos besoins. Il doit en être de même pour les institutions créées par les hommes. Dans ce cas-ci, c'est le recours aux sciences humaines qui est le plus en mesure d'aider la collectivité à solutionner les difficultés qu'elle rencontre. Les problèmes impliqués par la criminalité ne font pas exception.

29. WHITE, "The need for Cooperation Between the Legal Profession and the Psychiatrist in Dealing with the Crime Problem ", American J. of Psychiatry, vol. 7 (1927), p. 502. Citation reprise de J. Hall. : op. cit., p. 458.

30. Tous les déterministes acceptent cette idée selon laquelle la science n'a pas à intervenir dans le processus légal. Freud, dans le même ordre d'idées, dénonça le fait que la technique analyrique puisse servir à incriminer ou à disculper un accusé. Ferenczi, à la suire de Freud, suggéra de n'uriliser la ressource psychanalytique qu'après la condamnation du criminel. Lire là-dessus T. SZASZ, op. cit., pp. 135-1.38. 
Pour les déterministes réformistes, le criminel n'a pas à être jugé ni blâmé pour ses crimes. Le blâme tout comme l'incarcération dénotent une incompréhension des causes du comportement criminel et, pour cette raison, ne conduisent qu'à la récidive. Les sciences du comportement, pour leur part, sont en mesure (sinon aujourd'hui du moins dans un avenir prochain) de comprendre les causes du crime, de présenter un plan de réforme adapté aux besoins de réhabilitation du détenu et de prévenir efficacement la récidive. Mais pour cela, il faut changer radicalement le système judiciaire, dernier bastion de la totalité des erreurs entretenues sur la criminalité, sur ses causes et ses remèdes.

\subsection{Se passer de l'analyse de la responsabilité}

Les réformistes déterministes proposent d'oublier l'enquête de responsabilité du droit criminel et de limiter la tâche des juristes à la mise en évidence des causes matérielles du crime. Il s'agit donc de mettre en perspective la séquence des événements qui expliquent le crime et de juger la participation causale de l'inculpé à ces événements, sans avoir recours à l'autre type d'enquête causale concernant les motifs de l'agent. Les questions relevant de sa responsabilité et relatives aux conditions habituellement reconnues par le «mens rea» sont définitivement mises de côté et désignées comme des questions dépourvues de sens. Pour les déterministes, les juristes n'ont pas à tenter de résoudre l'insoluble, c'est-à-dire déterminer la responsabilité d'un inculpé par rapport à ses actions. Bien plus, cette tâche n'est même plus essentielle puisqu'une fois le «coupable » (en termes strictement causal) d'un crime identifié, les déterministes prévoient que des experts en sciences du comportement prendront la relève du processus judiciaire pour porter un «diagnostic » et proposer un «traitement» dans le but d'éviter la récidive et d'améliorer les chances de réhabilitation du criminel. Le but n'est donc pas en soi de punir de façon juste et équitable, mais «d'offrir » à chaque individu aux prises avec un problème de criminalité le meilleur traitement possible. L'attribution de la responsabilité est, à l'intérieur de ce processus, totalement court-circuitée.

Pour les déterministes, l'imputabilité telle que considérée par le droit criminel depuis ses origines est un artifice qui n'est pas du 
tout nécessaire à sa bonne marche, au contraire. Il s'agit par contraste pour une société comme la nôtre d'identifier clairement les objectifs prioritaires de l'appareil judiciaire (blâmer ou traiter) et de prendre les moyens pour y arriver. Les réformistes proposent l'intervention thérapeutique réhabilitative et généralisée :

The criminal court should cease with the findings of guilt and innocence, and the procedure thereafter should be guided by a professional treatment tribunal and be composed, say, of a psychiatrist, a psychologist, a sociologist or cultural anthropologist, an educator and a judge with long experience in criminals trials and with special interest in the protection of the legal rights of those charged with crime ${ }^{31}$.

Nous verrons cependant en conclusion que la cohérence interne des déterministes sur le problème philosophique de la responsabilité rencontre des difficultés qui, bien que différentes de la version médico-légale, n'en demeurent pas moins significatives du mal qu'ont ces chercheurs des sciences humaines à articuler leur propre connaissance autour de thèmes aussi fondamentaux pour la tradition philosophique.

\section{LA VERSION DE «RESPONSABILITÉ STRICTE »}

\subsection{Barbara Wootton : critique du réformisme pénal}

Cette dernière version du réformisme pénal et du problème de la responsabilité est associée à une personnalité majeure de l'histoire du réformisme pénal: Barbara Wootton.

Wootton est une juriste et sociologue britannique. Elle était considérée aux États-Unis et en Angleterre comme une des figures de proue du réformisme pénal, principalement à cause du radicalisme de ses positions sur la question ${ }^{32}$. Paradoxalement, c'est à partir de ses positions critiques vis-à-vis des tenants de la doctrine réformiste qu'on en vient à lui reconnaître une certaine autorité en la matière et à lui faire occuper une place particulière dans les débats

31. K. Menninger, op. cit., p. 139.

32. CF. les principales ceuvres de WOOTTON en la matière: Social Science and Social Patbology, op. cit.; Testament for Social Sciences: an Essay in the Application of Scientific Method to Human Problems, op. cit. et Crime and The Criminal Law: Reflection of a Magistrate and Social Scientist, London, Stevens \& Sons, 1963. 
entourant le réformisme. Ainsi, à tour de rôle, Wootton a critiqué chacune des deux versions antérieures du réformisme pénal sur le problème spécifique de la responsabilité. On peut résumer ses critiques de cette manière:

(i) L'entreprise d'élargir la règle de M'Naghten dans le but de lui donner une portée moins intellectualiste est une entreprise vouée à l'échec : « once we allow any movement away from a rigid intellectual test of responsibility on M'Naghten lines, our feet are set upon a slippery slope which offers no real resting place short of total abandonment of the whole concept of responsibility ${ }^{33}$. " Comme nous l'avons vu en première partie de cet article, Wootton croit avoir fourni les éléments principaux de cet échec de la version médico-légale (absence de critères indépendants, circularité des raisonnements, etc.).

(ii) La version déterministe est tout aussi fautive dans ses tentatives d'essayer d'imposer un cadre déterministe aux motivations profondes qui invitent à une réforme de nos pratiques judiciaires.

En fait, dans ce dernier cas, ce n'est pas la validité de la thèse déterministe en soi mais plutôt la place que les tenants de cette thèse cherchent à lui faire occuper que Wootton critique. $\grave{A}$ proprement parler, dit-elle, le recours à la thèse du déterminisme est superflu. Bien plus, il est préférable d'en faire l'économie afin d'éviter les discussions et les répliques « orthodoxes » entourant le débat sur la liberté humaine. L'opposition de Wootton au réformisme déterministe est donc plus formelle que réelle. Elle ne doute pas de la pertinence de la thèse déterministe sur le plan strictement philosophique, bien au contraire. Elle ne veut cependant pas en débattre et elle ne croit pas qu'il soit nécessaire de le faire pour justifier une réforme du type de celle proposée par les déterministes. En ce sens, l'argument de Wootton est essentiellement pragmatique : la vérité d'une thèse morale ou d'un système pénal comme celui que cherche à justifier l'auteure se trouve en dernière instance dans l'évidence positive des effets que les changements recommandés réussissent à apporter. L'appareillage argumentatif des déterministes

33. Social Science and Social Pathology, op. cit., p. 249. 
à l'égard de la responsabilité constitue un effort de trop et qui de surcroît les rendent très vulnérables à la réplique de ceux qui rejettent la thèse déterministe et qui sont prêts à leur livrer bataille sur cette question. Wootton affirmera qu'il est possible de faire l'économie de la thèse déterministe pour peu qu'on pose le problème de la responsabilité différemment.

\subsection{Dépasser le problème de la responsabilité : des jugements de « responsabilité stricte»}

"What the application of successful "mental medecine" to the treatment of anti-social persons demands is not determinism, but the ability to predict which persons are likely to be successfully treatable, and to devise methods that will deflect them from their unacceptable ways ${ }^{34}$.» Le système judiciaire que voudrait voir instauré Wootton constitue une tentative de généraliser une formule d'exception de nos pratiques actuelles du droit : les jugements de «responsabilité stricte» ${ }^{35}$. La "responsabilité stricte» est une forme de jugement qui ne s'applique habituellement dans le droit que pour une courte liste de délits où le législateur a décidé qu’à toute fin pratique, les conditions du «mens rea» ne devaient pas être sollicitées pour juger la culpabilité d'un agent. Dans les cas de " responsabilité stricte ", la présence de certains «éléments mentaux " lors du délit (intention, connaissance des conséquences immédiates de ses actions, etc.) n'est plus requise pour poser un verdict de culpabilité et pour sanctionner la peine appropriée. Les cas de négligence criminelle constituent un exemple typique de "responsabilité stricte ». Dans une cause de négligence criminelle, la question principale adressée au jury est de déterminer s'il reconnaît que l'accusé a effectivement commis la négligence qu'on lui reproche. On reconnaît peu de conditions d'excuses ${ }^{36}$. L'accusé

34. Ibid., p. 247.

35. L'expression anglaise «strict liability» a été traduite ici par " responsabilité stricte». J'emprunte cette traduction de la version française de The Concept of Law (Le concept de droit) effectuée par Michel van de Kerchove, Bruxelles: Publications des facultés universitaires St-Louis, 1976. Je reconnais par ailleurs que l'expression n'est pas exempte d'ambiguités.

36. Pour une étude cririque de la notion de " responsabilité stricte ", voir $R$. WASSERSTROM, "Strict Liability in the Criminal Law", Stanford Law Review, vol. 12 (1959-60), pp. 730-45. Repris dans G. EzORSKY (ed.), Pbilosophical Perspectives on Punishment, N.Y.: State University of New York Press, 1972, pp. 196-212. 
est reconnu « responsable » de sa négligence (si négligence il y a) et coupable devant la loi, indépendamment la plupart du temps de “l'esprit» ou des «raisons" pour lesquels il agissait. Le «mens rea " n'est plus nécessité et la préoccupation centrale du jury est de déterminer le déroulement des faits et la place qu'y occupe l'accusé.

En élargissant ces situations de « responsabilité stricte » à l'ensemble du droit criminel, Wootton veut nous transporter dans un univers pénal où les composantes cognitives et conatives de l'attribution de la responsabilité (le «mens rea») sont évincées complètement et non remplacées. Cette double éviction est similaire à l'approche préconisée par les déterministes à l'égard de la responsabilité dans le droit. Cela n'est pas sans raison. Wootton prétend qu'une approche de la criminalité qui privilégierait une intervention thérapeutique par rapport à une approche punitive peut à toute fin pratique se passer d'un verdict de responsabilité. Il faut néanmoins pour cela que soient clairement identifiés les nouveaux objectifs que nous voulons mettre en valeur à l'intérieur de notre système judiciaire. Le premier de ces objectifs est pour Wootton d'en arriver à un système qui soit le plus fonctionnel possible («workable system»). Wootton défend l'idée qu'il faut opter pour une procédure judiciaire la plus simple possible mais qui permettra néanmoins dans la majorité des cas une intervention thérapeutique généralisée à tous les individus reconnus coupables d'acte criminel. Dans cette voie, on comprendra qu'elle reconnaissait à la règle de M'Naghten certaines qualités : "The strength of the M'Naghten Rules lies, first and foremost, in the fact that by its very narrowness, it provides a safe and commonsensical definition of the minimum group about whose inclusion in the category of irresponsibles there can be no dispute ${ }^{37}$. " Ce que Wootton reproche à la règle n'est donc pas sa «fonctionnalité » mais, à la suite des tenants de la version médico-légale, son caractère restrictif. Pour cette raison, elle préfère à toutes les propositions déjà offertes un système qui (i) dépasserair franchement le problème de l'attribution de responsabilité, (ii) généraliserait les interventions thérapeutiques à l'ensemble de la population carcérale tout en (iii) simplifiant largement le processus judiciaire.

37. B. Wootton, “Diminished Responsibility: A Layman's View ", op. cit., p. 228. 
La réforme du système pénal que sollicite Wootton ressemble donc grandement à celle proposée par les déterministes. Il n'y aurait plus à l'intérieur du processus judiciaire la tâche de déterminer la responsabilité d'un individu face à un crime mais seulement celle de considérer les rapports de cause à effet entre différents événements physiques. Une fois établi ce rapport de cause à effet impliquant un agent, l'étape suivante consisterait à remettre le sort de celui-ci entre les mains de spécialistes du comportement qui auraient à choisir le meilleur traitement en vue de la réhabilitation du criminel: "Forget responsibility, and psychiatrists need no longer masquerade as moralists, but can return to their proper role as applied scientists [...] Forget responsibility and we can ask not whether an offender ought to be punished, but whether be is likely to benefit from punisbment ${ }^{38}$."

Wootton n'exclut pas la possibilité que ces spécialistes aient encore recours à des formes punitives plus conventionnelles, comme l'emprisonnement par exemple. Le recours à de telles techniques punitives peut se justifier à ses yeux si on évalue que pour un cas précis, c'est encore la meilleure façon de procéder à la réhabilitation d'un criminel. Ainsi, les techniques peuvent changer mais le projet réformiste fondamental demeure le même : réhabiliter le plus grand nombre de criminels. Son système a cet avantage, nous dit-elle, de se justifier d'après la qualité des succès obtenus. Ce sont les statistiques qui devraient en dernière instance nous y faire adhérer. La justification du remplacement de notre mode actuel de sanction par le système d'hygiène sociale de Wootton est, en dernier lieu, essentiellement pragmatique: elle procède de l'évaluation que l'on sera en mesure de faire des avantages indéniables à long terme de ce choix d'intervention auprès de la criminalité.

\section{CONCLUSION}

Ce survol des trois versions réformistes et de leur implication pour la problématique philosophique de la responsabilité nous a permis de faire un état de la question. Je compte maintenant conclure sur quelques remarques critiques à propos de difficultés théoriques que rencontrent chacun des trois projets présentés ici.

38. Ihid., p. 239. Je souligne. 
Ces critiques mettent sérieusement en doute la capacité des réformistes d'avoir pu présenter une position cohérente de la problématique de la responsabilité légale et morale.

\section{1. L'appareillage analytique nettement insuffisant des réformistes de la version médico-légale}

L'échec présumé de la version médico-légale à rendre compte de critères empiriques pour déterminer la responsabilité ou des degrés de responsabilité est symptomatique de la difficulté des théoriciens de ce programme de se former un point de vue suffisamment clair à propos de la notion de responsabilité ellemême. Leurs écrits sur cette question entretiennent d'énormes confusions. Ce qui manque à cette entreprise est tout d'abord une caractérisation plus complète du concept de responsabilité ${ }^{39}$. On aurait pu ainsi éviter des erreurs aussi simples que de confondre par exemple responsabilité causale (qui est en fait une autre façon de parler de causalité : « la tempête est responsable de la disparition de ce navire») et responsabilité attributive (qui est une notion beaucoup plus complexe de responsabilité comprenant des critères comme (i) les conditions mentales et psychologiques présentes lors de l'action, (ii) les rapports de causalité entre l'action et ses conséquences, (iii) l'éligibilité statutaire d'une personne à une sanction ${ }^{40}$. La conclusion: «expliquer n'est pas disculper », renvoyait à cette première considération sur la nature différente de ces deux types de responsabilité. De plus, comprendre la complexité du concept de responsabilité, c'est prendre en compte des thèses secondaires aussi importantes que la normativité du concept (on a besoin d'un cadre moral préexistant pour déterminer la responsabilité dans des situations précises) ${ }^{41}$, la distinction entre responsabilité légale et responsabilité morale ${ }^{42}$, ou encore la thèse de la «defeasability» des concepts légaux qui va, elle, jusqu'à mettre en cause

39. Comme T. Szasz l'a si bien montré dans La loi, la liberté et la psychiatrie, op. cit. Lire le chap. 10: «La responsabiliré pénale ».

10. Pour une caractérisation de ce type du concept de responsabilité, lire: H.L.A. HarT, "Postcript: Responsibility and Retribution", Punishment and Responsibility, op. cit., pp. $210-37$.

11. Voir là-dessus J.L. MaCkrt, "Responsibility and Language", Australian Journal of Philosophy, vol. 33 (1955), pp. 143-59.

12. Voir là-dessus Joël Flinhter(, «Problematic Responsibility in Law and Morals », op. cit. 
les tentatives d'en arriver à une caractérisation suffisante ${ }^{43}$. La définition de la responsabilité comme capacité de se conformer à une règle soulève des questions fondamentales qui ne trouvent pas leur réponse aisément. Elle sous-entend des considérations sur la nature de la responsabilité et sur son mode d'attribution qui ne sont discutées en général que superficiellement par les réformistes.

\section{2. L'égarement de certaines prétentions déterministes}

L'approche déterministe de la responsabilité et ses conséquences pour le réformisme pénal n'est pas non plus à l'abri de critiques sévères. La première de ces critiques s'adresse aux raisons motivant le recours à la thèse déterministe. C'est une réponse "classique » à un débat, il faut bien l'avouer, quelque peu suranné en philosophie... Cette réplique affirme que la théorie générale du déterminisme ne peut être inférée de cas particuliers ${ }^{44}$. Elle est une thèse métaphysique et non pas une conclusion scientifique comme le prétendent les réformistes. La science ne bénéficie pas d'une situation particulière qui lui permettrait de confirmer ou d'infirmer la véracité de cette thèse.

Par ailleurs, comme l'affirme J. Murphy ${ }^{45}$, le déterminisme prétendant qu'il est impossible pour la science de distinguer un meurtre d'un simple accident commet un abus de langage. Il existe quand même des différences substantielles entre un meurtre prémédité et froidement exécuté, et la mort d'une personne suite à un accident de chasse. Il appartient au droit et à la morale d'appliquer les règles nous permettant de distinguer ces différentes actions humaines et de nous aider à porter sur celles-ci un jugement, pas à la science.

43. C'est la thèse défendue par Hart dans "The Ascription of Responsibility and Rights", Proceedings of the Aristotelian Society, vol. 48-49 (1949), pp. 17 I-94.

44. Le déterminisme vu comme une thèse métaphysique évidemment. Voir là-dessus la nute 24. Les réformistes tuut comme Murphy ne font pas la distinction de statut entre principe de causalité universelle er déterminisme, ce qui permet de justifier la portée de la critique de Murphy.

45. JG. MurPhy, Retribution, Justice and Therapy, Dordrecht: D. Reidel Publishing Company, 1979. L'ouvrage de Murphy se veut un essai critique et d'influence kantienne du réformisme pénal. 
Dans un autre ordre d'idées, le réformisme déterministe, comme j'ai déjà pu l'indiquer ailleurs, assume trop rapidement l'incompatibilité entre déterminisme et responsabilité. Cette seule question constitue un problème complexe caractéristique des débats à finir en philosophie de l'action contemporaine. Dans cette voie on pourrait développer une stratégie de réplique au déterminisme similaire à celle que l'on retrouve en philosophie du droit sur ces questions. H.L.A. Hart, par exemple, croit que certaines approches de la responsabilité légale ne sont pas nécessairement en contradiction avec des formes du déterminisme en autant que ces dernières reconnaissent deux principes:

(i) Le déterminisme ne peut nier :

- que certaines affirmations du genre « ceci est le résultat de son choix » ou « il en est advenu selon ce qu'il avait choisi », ne sont pas dépourvues de sens;

- que nous sommes en général satisfaits lorsque nos choix se concrétisent;

- que nous sommes capables de prédire avec un certain succès les résultats à court terme de nos choix.

(ii) De plus, il ne peut affirmer :

- que nous connaissions déjà les lois dont il dit «qu'elles peuvent ou qu'elles doivent exister »;

- que ces lois soient suffisamment simples en ellesmêmes pour être découvertes par un être humain; ou

- que ces lois soient suffisamment simples pour qu'un être humain puisse en faire usage afin de prédire sa propre conduite ou la conduite des autres ${ }^{46}$.

Les propositions de Hart interpellent le déterminisme sur deux de ses présuppositions: (i) les conditions minimales pour construire une théorie de la responsabilité cobérente (l'accent n'est plus sur la notion de liberté mais sur l'existence empirique de l'expérience du choix, sur le côté proprement fonctionnel de ce pouvoir et sur la valeur sociale que nous lui accordons; (ii) la

46. Nous trouvons ces propositions de Hart aux pages 28 et 29 de Punishment and Responsibility, op. cit. Je traduis. 
nature, la portée et l'usage des lois du comportement bumain (le fossé qui sépare l'acceptation de l'existence de telles lois et le cadre d'une application éventuelle). La « sagesse » et surtout la prudence qui nous demandent de souscrire, du moins dans l'état actuel de nos connaissances, aux principes invoqués par Hart mettent la responsabilité légale à l'abri d'un certain «déterminisme dur » et aux prétentions trop larges qui ignore ou du moins sous-estime la complexité des fonctions dévolues au principe de responsabilité dans une société.

\section{3. Les fondements moraux de la responsabilité}

Wootton reconnaissait déjà certaines limites à son système d'hygiène sociale ${ }^{47}$. D'abord, tout succès de nos techniques réhabilitatives, si grand soit-il, demeure indépendant de la force dissuasive d'un tel système auprès de l'ensemble de la population. L'approche d'hygiène sociale de Wootton n'assure pas de la même façon qu'un droit plus répressif l'observation des règles par la majorité. L'autre limite reconnue par Wootton se rapporte aux critères d'efficacité et de fonctionnalité. Le pragmatisme de sa position ne réussit pas à épuiser toute considération morale. Par exemple, la meilleure façon d'éviter la récidive est encore la peine de mort : elle a un taux de réussite de $100 \%$ ! Dans le même ordre d'idées on pourrait argumenter qu'il est plus raisonnable d'exécuter un psychotique qu'un individu sain, puisqu'il est habituellement reconnu que le dernier répond mieux à certains traitements réhabilitatifs! Le critère de l'efficacité, dans ces cas et pour bien d'autres, doit pouvoir être limité par des valeurs qu'il reste encore à établir dans son système et dans celui des déterministes. Mais ce problème de la « moralité » du système est inhérent, selon Wootton, à l'usage de toutes techniques scientifiques pouvant influencer le destin de l'homme. Cela n'interdit en rien, selon elle, d'avancer plus à fond dans son programme d'hygiène sociale.

Le radicalisme de Wootton et des déterministes à l'égard d'un système de "responsabilité stricte» nous invite ainsi à poser le problème fondamentai de la responsabilité et de sa justification

47. Voir Social Science and Social Pathology, pp. 252 et 253. 
dans notre système légal. C'est donc sur cette dernière question que je terminerai mes commentaires critiques: que perdrionsnous dans un système de responsabilité stricte?

Je compte puiser les éléments de réponse à cette dernière mise en doute chez H.L.A. Hart. Je considère Hart comme étant probablement le philosophe qui a le mieux réussi à circonscrire le rôle et l'importance de la responsabilité dans nos structures juridiques, institutionnelles et morales. Ses considérations sur le réformisme pénal fournissent selon moi les éléments essentiels de toute discussion critique sur le sujet. C'est ainsi que pour saisir l'importance de la prise en compte de certains éléments mentaux à l'intérieur du processus légal menant à la condamnation, Hart nous suggère une analogie avec certaines formes de transactions civiles sanctionnées par le droit ${ }^{48}$. Il est habituellement reconnu que ces transactions (vente, testament, mariage) doivent s'effectuer entre gens libres et consentants si l'on désire qu'elles soient reconnues valides. Le cas échéant, ces transactions peuvent être annulées à n'importe quel moment. La présence de ces « éléments mentaux » chez les participants à ces transactions est donc considérée comme nécessaire à leur reconnaissance ultérieure. Cette exigence selon laquelle toute transaction doit être accompagnée de certains éléments mentaux comporte deux avantages :

(i) celui, pour l'individu qui choisit librement de passer un contrat avec autrui, de permettre de déterminer ce que le futur sera (d'augmenter son pouvoir de prédiction);

(ii) celui de lui permettre de déterminer ce futur avec l'appui coercitif de la loi afin que ses choix soient respectés (d'améliorer l'efficacité de ses choix) ${ }^{49}$.

Si on transpose maintenant ces conditions de validité à notre système judiciaire, on peut et on doit s'attendre à des garanties semblables. L'attention accordée à la présence ou à l'absence d'éléments mentaux avant d'établir un verdict de responsabilité nous assure que certaines actions que nous pourrions poser contre

48. On pourra lire à ce sujet «Legal Responsibility and Excuses», Punishment and Responsibility, op. cit., pp. 40-50 et «The Ascription of Responsibility and Rights", op. cit.

49. Ibid., p. 45 et suivantes. 
notre gré ou sans en connaître la véritable nature ne seront pas retenues contre nous. Nous n'en porterons pas la responsabilité. Dans le cas qui nous intéresse, cela a comme avantage de minimiser les interventions de l'État et de maximiser le potentiel des libertés individuelles de chaque membre de la société. Chez Hart, ces libertés individuelles sont reconnues comme limitées mais aussi assurées par les institutions civiles. Les conditions atténuantes ou «excusantes" sont une garantie (pas à elles seules, bien sûr) de protection de la liberté des individus contre les abus possibles de la société et de « l'intérêt du bien commun ». Les systèmes réformistes offrent peu de garanties à ce niveau, bien au contraire. Ils peuvent conduire à une prophylaxie pénale démesurée et difficile à contrôler.

Hart propose de considérer l'action humaine comme un système de choix. Une entité que les institutions sociales devraient reconnaître et protéger. Évidemment ajoute-t-il, choisir un système pénal où l'importance de la responsabilité est reconnue c'est aussi accepter de prendre un « risque social » plus élevé. En effet, s'il est vrai que le «mens rea» renforce l'auto-contrôle, il ne le garantit pas:

If we contrast our system with one in which men were conditioned to obey the law by psychological or others means, or one in which they were liable to punishment or « treatment » whether they had volontarily offended or not, it is plain that our system takes a risk which these alternatives systems do not. Our system does not interfere till harm has been done and has been proved to have been done with the appropriate "mens rea". But the risk that is here taken is not taken for nothing. It is the price we pay for general recognition that a man's fate should depend upon his choice and this is foster the prime social virtue of selfrestraint $[\ldots]$ Human society is a society of persons ; and persons do not view themselves or each other merely as so many bodies moving in ways which are sometimes harmful and have to be prevented or altered. Instead persons interpret each other's movements as manifestations of intention and choices, and these subjective factors are often more important to their social relations than the movements by which they are manifested or their effects ${ }^{50}$.

On peut tirer deux conclusions de cette citation. Il est tout d'abord normal que, jusqu'à un certain niveau, les règles que nous

50 Ibid., p. 182. 
utilisons à l'intérieur du droit reflètent celles qui nous servent à établir nos jugements moraux. Le contraire serait inquiétant. S'il est un lien, aussi mince soit-il, que droit et moralité peuvent entretenir, c'est certainement celui de s'emprunter mutuellement les concepts-clés de leur langage respectif (la responsabilité, la culpabilité, la négligence, l'innocence, etc.). Un système légal où les jugements de «responsabilité stricte» sont généralisés ne rencontre en rien l'usage normal du langage moral, bien au contraire, il le dissout ${ }^{51}$. La question soulevée par Hart est donc très pertinente: est-il possible que nos institutions reflètent un autre langage que celui que nous utilisons pour former nos jugements moraux? Ceci va plus loin que de souligner certains écarts qui peuvent toujours se produire entre les règles que contiennent un registre de lois et celles, différentes, que nous pouvons adopter pour diriger notre conduite. La question pose l'éventualité qu'un concept aussi important que celui de responsabilité puisse s'éteindre entièrement dans notre système légal tout en demeurant encore présent, courant et actif à l'intérieur du langage moral de nos pratiques quotidiennes. Comment l'usage de cette expression pourrait-elle survivre après que l'on ait effacé du droit la valeur que nous lui accordons comme individu et comme société?

La deuxième conclusion concerne la distinction que le concept de responsabilité nous permet de faire entre certaines actions. Cette distinction entre plusieurs actions ne délimite pas seulement notre cadre moral mais aussi toute notre vie sociale ainsi que notre façon d'entrer en contact avec les autres. Si on me frappe ou encore si on me jette un regard désapprobateur, il est certain que ma conduite ultérieure sera influencée par le sens que j’accorde à ces actions envers moi. Comment pourrait-elle l'être et de quelle manière si je ne pouvais plus accorder de sens à des expressions comme « intentionnellement », «par accident », « volontairement »? Quelle serait mon attitude vis-à-vis des individus ayant posé ces gestes? Sur quelle base serait-il possible pour moi d'entrer en contact avec eux? Wootton ne contredirait probablement pas le fait que la responsabilité engendre des conséquences pour la

51 C'est l'argument principal servi à l'encontre des partisans de la «strict-liability» dans le droit. Voir R. WASSERSTROM, op. cit. 
formation d'un tissu social, mais son système tout comme celui des autres versions réformistes en général, n'accepte pas de donner la place que le concept s'est mérité dans le cadre de l'organisation, la compréhension et ultimement la formation de nos vies.

En définitive, on peut dire du concept de responsabilité qu'il est le corollaire pour notre vie en société de ce qu'est le concept de causalité pour notre compréhension de l'univers: "Cause is the ciment of universe; the concept of cause is what holds together our picture of the universe, a picture that would otherwise disintegrate into a diptych of the mental and the physical 52. "Il faut donc formuler l'hy pothèse avec Hart qu'au croisement de la philosophie morale et de la philosophie de l'action, il existe une caractérisation possible du concept de responsabilité qui tienne compte de la nature essentiellement pratique et nécessaire de l'ascription de responsabilités. C'est dans un programme de recherche en philosophie de l'action qui examine l'action humaine sous l'angle de ses conséquences plutôt que comme simple produit d'un agent que jestime possible d'élaborer cette caractérisation. Les contributions récentes en philosophie de l'action ne nous ont pas permis de comprendre suffisamment le rôle que peut jouer la responsabilité comme mécanisme d'évaluation de nos comportements et peutêtre, faut-il en faire une seconde hyporhèse, nous ont-elles éloigné plus qu'elles nous on rapproché de la véritable nature du langage d'action.

Département de philosophie

Université du Québec à Montréal

52. Donald Davinson, Essays on Action and Events, Oxford: Oxford Universicy Press, 1980, p. xi 